\title{
CORRELATION BETWEEN MAGNETIC AND ELECTRONIC PROPERTIES OF $\mathrm{Sn}_{1-x} \mathrm{Gd}_{x} \mathrm{Te}^{*}$
}

\author{
M. Górska, T. Story, M. Arciszewska, E. Grodzicka, Z. Golacki \\ AND A. LUSAKOWSKI
}

Institute of Physics, Polish Academy of Sciences

Al. Lotników 32/46, 02-668 Warszawa, Poland

\begin{abstract}
Magnetic susceptibility, electron paramagnetic resonance and transport properties of $\mathrm{Sn}_{1-x} \mathrm{Gd}_{x} \mathrm{Te}$ with $0.04<x<0.07$ and hole concentrations in the range from $0.7 \times 10^{20}$ to $16 \times 10^{20} \mathrm{~cm}^{-3}$ were investigated. After annealing of the $\mathrm{Sn}_{1-x} \mathrm{Gd}_{x}$ Te samples with $x<0.05$ in $\mathrm{Sn}$ vapor their hole concentration decreased from $5 \times 10^{20} \mathrm{~cm}^{-3}$ to about $3 \times 10^{20} \mathrm{~cm}^{-3}$ and their paramagnetic Curie temperature increased a few times. In samples with $x>0.05$ no significant change in the magnetic properties was observed after annealing, even at lower hole concentrations. The results can be explained by assuming that an indirect exchange interaction, $4 f-5 d$-band electrons, is responsible for the coupling among Gd ions.
\end{abstract}

PACS numbers: 75.20.Ck, 75.30.Et

\section{Introduction}

Magnetic properties of Bridgman-grown $\mathrm{Sn}_{1-x} \mathrm{Gd}_{x} \mathrm{Te}$ have been investigated previously $[1,2]$. The results showed a very weak antiferromagnetic exchange coupling among Gd ions, with no evidence for RKKY-type ferromagnetic interaction observed in $\mathrm{Sn}_{1-x} \mathrm{Mn}_{x} \mathrm{Te}$ [3] and $\mathrm{Pb}_{1-x-y} \mathrm{Sn}_{y} \mathrm{Mn}_{x} \mathrm{Te}$ [4]. However, in the as-grown samples the Gd content and carrier concentration were nearly inversely proportional. Here we are reporting measurements of magnetic properties of ${S n_{1-x}}_{G_{d}} \mathrm{Te}$, for a series of values of $x$, with carrier concentration varying over an order of magnitude for a given $\mathrm{Gd}$ content.

*This work was supported in part by the M. Sklodowska-Curie Joint Fund II, No. PAN/NSF-92-113 and by the State Committee for Scientific Research (Republic of Poland) grant No. 204829101. 


\section{Experiment}

The samples of $\mathrm{Sn}_{1-x} \mathrm{Gd}_{x} \mathrm{Te}$ were cut from a boule grown by the Bridgman technique. The nominal $x$ values varied from 0.05 to 0.09 . The samples were annealed isothermally at about $700^{\circ} \mathrm{C}$ in $\mathrm{Sn}$ or Te atmosphere, in order to reduce or increase their carrier concentration, respectively.

The carrier concentrations and Hall mobilities were determined by standard Hall effect and conductivity measurements over a temperature range from 4.2 to $300 \mathrm{~K}$, with an accuracy of about $15 \%$. All the samples were $p$-type. The hole concentrations were in the range from $0.7 \times 10^{20}$ to $16 \times 10^{20} \mathrm{~cm}^{-3}$ and the mobilities from 4 to $300 \mathrm{~cm}^{2} /(\mathrm{V} \mathrm{s})$ at $77 \mathrm{~K}$.

The magnetic susceptibility was measured by the ac mutual inductance technique in alternating magnetic fields up to $40 \mathrm{Oe}$, over a temperature range from 1.5 to $70 \mathrm{~K}$.

\section{Results and discussion}

The susceptibility data were fitted over the temperature range from 10 to $70 \mathrm{~K}$ to the Curie-Weiss law. The Curie constant and paramagnetic temperature, $\theta$, were fitting parameters. The diamagnetic susceptibility of the host lattice was taken from the susceptibility measurements of SnTe as $-5 \times 10^{-7} \mathrm{emu} / \mathrm{g}$. The effective content of Gd ions, $\bar{x}$, i.e. the content in the cation sublattice of magnetic ions with a spin equal to $7 / 2$, and the nearest neighbor exchange parameter $J / k_{\mathrm{B}}$ ( $k_{\mathrm{B}}$ is the Boltzmann constant), were determined from the Curie constant and $\theta$ as described in Ref. [2], with estimated errors of about $20 \%$. The effective Gd content was usually smaller than the nominal one, as in other diluted magnetic semiconductors.

In Fig. 1 we show the paramagnetic Curie temperature over the effective Gd content, $\theta / \bar{x}$, as a function of the carrier concentration for different samples. We see that for crystals with $\bar{x}<0.05, \theta / \bar{x}$ at $p \approx 3 \times 10^{20} \mathrm{~cm}^{-3}$ is a few times larger than at $p>5 \times 10^{20} \mathrm{~cm}^{-3}$. In these samples we also observed a characteristic cusp at about $1.6 \mathrm{~K}$ in the temperature dependence of the magnetic susceptibility, which is an evidence of a spin glass phase. The hole concentration could not be reduced below $3 \times 10^{20} \mathrm{~cm}^{-3}$ by further annealing. In samples with $\bar{x}>0.05$ the hole concentration could be reduced below $10^{20} \mathrm{~cm}^{-3}$, but no significant increase in $\theta$ with decreasing $p$ was observed, and no cusp in the susceptibility appeared. In addition, the hole mobilities at $p \approx 3 \times 10^{20} \mathrm{~cm}^{-3}$ were almost an order of magnitude higher in samples with $\bar{x}<0.05$ than in samples with $\bar{x}>0.05$. The EPR measurements revealed the $g=2.0$ resonance for all samples.

The experimental findings presented above can be explained by a model assuming that the Gd $5 d$ energy state is degenerate with the valence band of $\mathrm{Sn}_{1-x} \mathrm{Gd}_{x} \mathrm{Te}$. Schematic diagram of the energy levels and densities of states in samples with $\bar{x}<0.05$ is shown in Fig. 2. The exchange coupling among Gd ions is due to an intraatomic $4 f-5 d$ exchange and interatomic $5 d-5 d$ exchange via conducting holes, and is inversely proportional to $E_{5 d}-E_{\mathrm{F}}$ [5]. Since $\theta$ is proportional to the sum of exchange constants over the crystal, the resonant enhancement of $\theta$ 
in samples with $\bar{x}<0.05$ at lowest hole concentration may be caused by $E_{\mathrm{F}}$ approaching $E_{5 d}$. That confirms the proposed mechanism of exchange interaction.

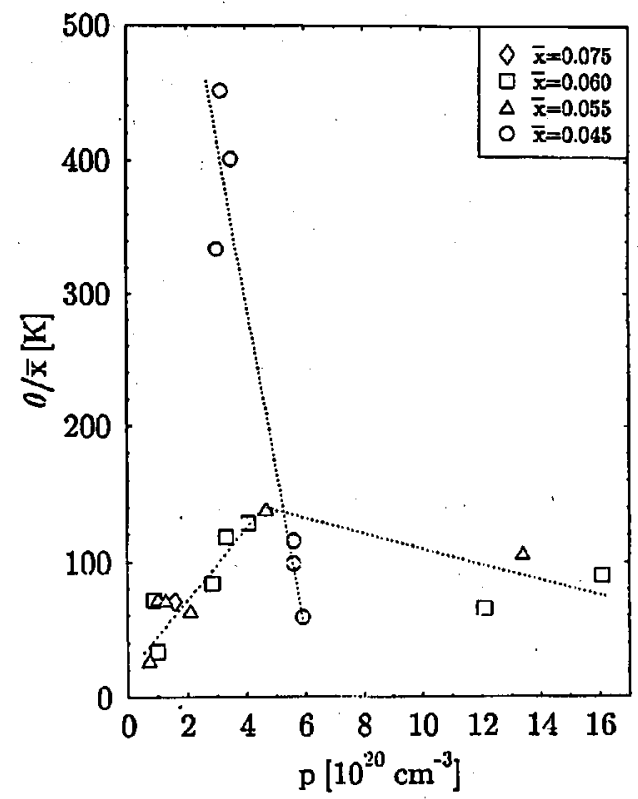

Fig. 1. $\theta / \bar{x}$ vs. hole concentration in $\mathrm{Sn}_{1-x} \mathrm{Gd}_{x}$ Te. Each $\bar{x}$ is the effective Gd content determined from susceptibility measurements and averaged over few samples, with an estimated error \pm 0.004 . Dotted lines are a guide to the eye.

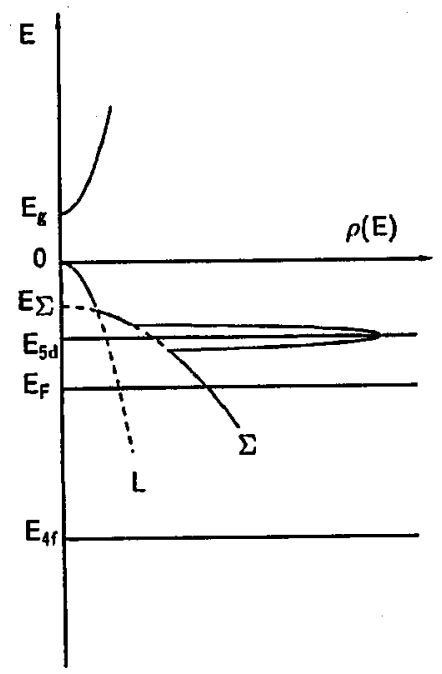

Fig. 2. Schematic diagram of energy levels and densities of states in $\mathrm{Sn}_{1-x} \mathrm{Gd}_{x} \mathrm{Te}$. 
We believe that in samples with $\bar{x}>0.05$ the Gd $5 d$ level is degenerate only with the light hole $L$-band, the Fermi level is far below the Gd $5 d$ level and practically all $\mathrm{Gd}$ ions are ionized $\mathrm{Gd}^{3+}$ ions with the outer electron configuration $4 f^{7}$. The Fermi level may then raise relatively high in the valence band, resulting in the low hole concentration, but is still too far from the Gd $5 d$ level to cause a significant increase in the exchange coupling. In samples with $\bar{x}<0.05$ the $5 d$ energy state is degenerate both with the heavy hole $\Sigma$-band and the light hole $L$-band, as shown in Fig. 2. The Fermi level may then move close to the Gd $5 d$ level, causing the resonant enhancement in $\theta$. A significant fraction of Gd ions would then be $\mathrm{Gd}^{2+}$ ions, with the outer electron configuration $4 f^{7} 5 d^{1}$. Since no significant dependence of $\bar{x}$ on hole concentration was observed, the $5 d$ level is probably delocalized, and the magnetic moment of $\mathrm{Gd}$ in both charge states is $7 / 2$, related only to the half-filled $4 f$ shell.

\section{Acknowledgments}

We are grateful to Prof. R.R. Gałazka and to Prof. J.R. Anderson for many helpful discussions.

\section{References}

[1] H.T. Sa vage, J.J. Rhyne, in: Magnetism and Magnetic Materials-1971, Chicago, Ed. D.C. Graham, J.J. Rhyne, AIP Conf. Proc. No. 5, American Institute of Physics, New York 1972, p. 879.

[2] M. Górska, J.R. Anderson, G. Kido, S.M. Green, Z. Gołacki, Phys. Rev. B 45, 11702 (1992).

[3] W.J.M. de Jonge, T. Story, H.J.M. Swagten, P.J.T. Eggenkamp, Europhys. Lett. 17, 631 (1992).

[4] T. Story, G. Karczewski, L. Świerkowski, R.R. Gałązka, Phys. Rev. B 42, 10477 (1990).

[5] A. Łusakowski, to be published. 